\title{
FidélISATION DU COUPLE HÔte-VECTEUR FACTEUR PROBABLE DE SPÉCIATION : 14 espèces de Plasmodium de la Pie
}

\author{
CHAVATTE J.M.*, CHIRON F.**, CHABAUD A.* \& LANDAU I.*
}

Summary: PROBABLE SPECIATIONS BY "HOST-VECTOR "FIDÉLISATION": 14 SPECIES OF PLASMODIUM FROM MAGPIES

33 Magpies resident in two parks close to Paris were investigated for the presence of Plasmodium parasites. The majority of the birds were found to be infected with multiple parasite species. A total of 14 species were observed, and of these 10 were novel and consequently described, and two could not be assigned with confidence. It is hypothesized that the unexpected abundance of species is due to a phenomenon which we term "host-vector 'fidelisation'". Indeed, the combination of the eco-biological characteristics of the host (mating pairs in contiguous, but strictly defined territories) with those of the vector (numerous Aedes species with distinct behavior), would generate fragmentation of the niches. This type of isolation overlays others known for parasitic populations (geographical, circadian, microlocalisations), leading to the formation of independent host-parasite niches which in turn lead to speciation.

KEY WORDS : Plasmodium, new species, Pica pica, periurban park, multiple intra-genus speciation, host-vector "fidélisation".

\section{INTRODUCTION}

L 'examen du sang de Pies, Pica pica (L., 1758) de deux parcs périurbains du nord de Paris a révélé 1 l'existence inattendue de 14 espèces différentes de Plasmodium. Presque toutes les Pies sont infectées et, dans la presque totalité des cas, il s'agit d'infections mixtes par plusieurs espèces. Une même Pie peut héberger jusqu'à sept espèces différentes de Plasmodium. En analysant les conditions particulières de nos deux lieux de prospection, nous sommes conduits à accorder une importance particulière à une spéciation secondaire à un isolement par fidélisation du couple hôte-vecteur.

\section{MATÉRIELS ET MÉTHODES}

\section{Matériel BIOLOGIQUe}

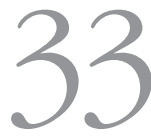

Pies ont été capturées dans le cadre d'un programme de recherches sur le fonctionnement et la dynamique de cette espèce. Les

* Parasitologie comparée et modèles expérimentaux, USM 307, Muséum national d'histoire naturelle, CP 52, 61, Rue Buffon, 75231 Paris Cedex 05, France.

*** Conservation des espèces, restauration et suivi des populations, UMR 5173, Muséum national d'histoire naturelle, CRBPO. CP 51, 55, rue Buffon, 75005 Paris, France.

Correspondance : Irène Landau.

Tél. : + $33(0) 140793500$ - E-mail : landau@mnhn.fr

\section{Résumé :}

Les Plasmodium de 33 Pies établies dans deux parcs proches de Paris ont été étudiés. La plupart des Oiseaux étaient polyparasités: 14 espèces dont 10 nouvelles ont été observées et décrites. L'abondance surprenante d'espèces différentes est attribuée à un phénomène qui est désigné sous le terme de "fidélisation du couple hôte-vecteur". En effet, les particularités biologiques de I'hôte (territoires contigus, mais bien définis) et des vecteurs présumés (nombreuses espèces d'Aedes à biologie distincte) entrainent un morcellement des niches. Ce type d'isolement s'ajoute aux autres facteurs classiques en parasitologie (géographie, microlocalisations, horaires) et aboutit à des isolements multiples, donc à de nombreuses spéciations.

MOTS CLÉS : Plasmodium, nouvelle espèce, Pica pica, Aedes, parc périurbain, spéciation intra-générique multiple, fidélisation hôte-vecteur.

captures ont été effectuées par le Centre de recherches sur la biologie des populations d'oiseaux (CRBPO) du Muséum national d'histoire naturelle de Paris. Les Pies proviennent de deux grands parcs périurbains, situés au nord-est de Paris : le Parc de la Courneuve $-\mathrm{N} 48^{\circ}$ $57^{\prime} 21^{\prime \prime} / \mathrm{E} 02^{\circ} 24^{\prime} 20^{\prime \prime}$ - et le Parc du Sausset - N $48^{\circ}$ $57^{\prime} 28^{\prime \prime} / \mathrm{E} 02^{\circ} 30^{\prime} 59^{\prime \prime}$-, s'étendant respectivement sur 400 ha pour le premier et 200 ha pour le second. 26 Pies, ont été capturées d'avril à juin 2005 et sept en avril 2006, et certaines ont été maintenues en captivité, puis relâchées en fin d'étude.

En plus des Plasmodium, des infections fréquentes par Haemoproteus Kruse, 1890 et peu fréquentes par Leucocytozoon Berestneff, 1904, Trypanosoma Gruby, 1843, et Lankesterella Labbé, 1899, ont été observées.

\section{MÉTHODES D'ÉTUde}

Un prélèvement sanguin est fait dans la veine brachiale et, pour certaines pies, plusieurs prélèvements espacés de quelques heures à plusieurs semaines sont effectués. Les frottis sont fixés par le méthanol $100 \%\left(\right.$ Merck $\left.^{\circledR}\right)$ et colorés pendant une heure par le colorant de Giemsa $\left(\right.$ Merck $\left.^{\circledR}\right)$ dilué à $10 \%$ dans du tampon phosphate ( $\mathrm{pH}=7,4)$. Les lames ne sont examinées qu'après avoir été montées à l'Eukit ${ }^{\circledR}$ pour éviter la décoloration du frottis par l'huile à immersion. 
Des échantillons de sang sont cryopréservés à $-20^{\circ} \mathrm{C}$ directement ou à $-80^{\circ} \mathrm{C}$ dans une solution $\mathrm{v} / \mathrm{v}$ de DMSO $\left(\right.$ Sigma $\left.{ }^{\circledR}\right)$ à $20 \%$ pour une analyse moléculaire ultérieure.

\section{MATÉRIEL TYPE}

En raison du polyparasitisme, nous avons rejeté la désignation d'hapantotypes et choisissons pour chaque espèce un holotype constitué par un schizonte caractéristique de l'espèce, clairement marqué par un cercle gravé au diamant sur la lamelle du frottis, et photographié sur la planche 15. Ces types sont déposés au Muséum national d'histoire naturelle de Paris, dans la collection du laboratoire de Parasitologie comparée et modèles expérimentaux, sous les numéros $438 \mathrm{LV}$, tiroir PXIII, 51 à 60 . Les auteurs des nouveaux taxa sont Chavatte et Landau.

\section{DESCRIPTIONS}

\section{CRITÈRES D'IDENTIFICATION \\ ET PRINCIPAUX CARACTÈRES MORPHOLOGIQUES}

$\mathrm{N}$ ous avons utilisé les critères morphologiques classiques (cf. Garnham, 1966; Valkiunas, 2005) : forme des gamétocytes; taille des schizontes; abondance du cytoplasme; nombre et distribution des noyaux, et en particulier dispersion au sein du cytoplasme ou bien, bourgeonnement périphérique; caractères du pigment; position dans la cellule-hôte; effet sur le globule rouge.

Les gamétocytes arrondis ont été faciles à rattacher aux quatre espèces correspondantes. Par contre, les gamétocytes allongés ont été plus difficiles à identifier du fait du nombre élevé d'espèces. Les identifications proposées s'appuient sur leur coexistence avec les espèces présentes chez le même individu et avec les principaux éléments morphologiques : taille, forme, place et effet sur l'hématie.

L'analyse morphologique des gamétocytes confirme que les observations de Hawking et al., 1969, sur l'évolution du pigment de P. gallinaceum Brumpt, 1935, parasite de la Poule, se vérifiaient chez toutes les espèces, que les gamétocytes soient ronds ou allongés. Le pigment est d'abord rassemblé en grains à une extrémité (gamétocytes allongés) ou près d'un bord (gamétocytes ronds), puis se déplace vers le milieu pour se disperser. Il s'agglomère ensuite en plusieurs masses dans le gamétocyte vieillissant. Nous n'avons figuré cette évolution que pour $P$. bioccai n. sp. et $P$. dorsti n. sp. décrits ci-dessous.

Il est important de noter que les caractéristiques morphologiques ne sont observables que sur des infections modérées $(0,5 \%$, au maximum). Au-dessus de cette parasitémie, la morphologie est altérée et le diagnostic devient aléatoire. Au cours de ce travail nous nous basons essentiellement sur les descriptions originales. Certaines descriptions originales correspondent manifestement à des infections mixtes et les redescriptions et les synonymies sont hasardeuses, a fortiori lorsque l'hôte et la région géographique ne correspondent pas à celles du matériel type.

\section{DESCRIPTIONS MORPHOLOGIQUES DES ESPÈCES DE Plasmodium Détectées Chez les Pies}

\section{PLASMODIUM GHADIRIANI N. SP. (planche 3, figures 1-10)}

L'espèce est dédiée à Esmail Ghadirian.

Matériel holotype sur la lame : $356 \mathrm{LV}$; planche 15, figure 3; Collection : $438 \mathrm{LV}$, PXIII, 55.

Le jeune schizonte à 2-3 noyaux est en position latérale le long du noyau du globule rouge (GR) dont il dépasse souvent un pôle. De forme allongée, incurvée, dodue, occupant parfois presque tout l'espace entre noyau et membrane du GR, son cytoplasme bleu est abondant et contient quelques vacuoles blanches. Le pigment est noir en grains de petite taille et situé à une extrémité.

En grandissant, le schizonte s'allonge, en boudin. Au début il contient trois à six masses chromatiniennes assez grandes, denses, irrégulières et espacées dans un cytoplasme bleu abondant, l'ensemble évoquant un début de cytomérisation. Les masses chromatiniennes occupent de plus en plus de volume puis, se fragmentent et finissent par former, dans le schizonte âgé, 18 à 24 noyaux régulièrement répartis dans le cytoplasme. Le schizonte âgé occupe tout l'espace entre noyau et bord du GR. Il dépasse un pôle du noyau de l'hématie, peut être incurvé à ce niveau et atteint ou dépasse légèrement le pôle opposé.

Le GR est légèrement élargi et son noyau, est fréquemment repoussé latéralement par les formes âgées. Le pigment, parfois dispersé chez le schizonte jeune, se rassemble en amas de plusieurs grains parfois agglomérés en une masse noire.

Comme les schizontes, les gamétocytes sont allongés entre noyau et bord du GR, repoussant le noyau de la cellule hôte. Ils sont d'abord droits, courts, avec un pigment rassemblé à une extrémité, puis plus dodus, avec une extrémité un peu rétrécie et l'autre élargie dépassant le noyau du GR et légèrement inclinée à ce niveau. Les gamétocyte et les schizontes âgés contiennent de nombreuses petites vacuoles.

- Diagnostic différentiel

Les différences avec $P$. tranieri n. sp. sont traitées cidessous avec cette espèce.

$P$. ghadiriani doit être différencié des espèces ayant comme lui de grands schizontes et des gamétocytes allongés. 
Il diffère de $P$. lophurae Coggeshall, 1938, parasite de Lophura ignita (Shaw, 1798), redéfini par Grès et Landau, 1997, et de P. bigueti Landau et al., 2003, parasite de Passer domesticus (L., 1758) par le nombre plus élevé de noyaux (18-24 contre 14 au maximum pour $P$. lophurae et $P$. bigueti).

$P$. ghadiriani diffère de $P$. circumflexum Kikuth, 1931, parasite de Turdus pilaris L., 1758 par la forme générale de ses gamétocytes et schizontes, qui sont seulement incurvés à une extrémité alors que ceux de $P$. circumflexum ont tendance à entourer le noyau du GR. Enfin, l'espèce la plus proche morphologiquement est P. fallax, parasite de Syrnium nuchale (= Strix woodfordii nuchalis) (Sharpe, 1870) décrit par Schwetz, 1930 et redéfini par Garnham, 1966, à partir du matériel de Schwetz. Les deux espèces diffèrent par le nombre de mérozoïtes (18 à 24 pour P. ghadiriani, "jusque 16", selon Schwetz, pour P. fallax). P. fallax diffère également de notre espèce par le caractère amiboïde des jeunes schizontes qui envoient des filopodia dans le GR, la présence "caractéristique" selon Garnham d'une ou deux conspicous : grandes vacuoles blanches au lieu des nombreuses petites vacuoles de $P$. ghadiriani.

Selon Schwetz, dans sa description de P. fallax, "ce qui distingue, avant tout, les formes en division de notre espèce de toutes les autres espèces connues, c'est leur forme allongée en vrai croissant dont les extrémités englobent les extrémités du noyau du globule rouge", ce qui n'est pas le cas de P. ghadiriani. Enfin, les gamétocytes et les schizontes de notre parasite déplacent le noyau du GR, ceux de P. fallax non.

\section{PLASMODIUM TRANIERI N. SP.} (planche 4, figures 1-10)

L'espèce est dédiée à Michel Tranier.

Matériel holotype sur la lame : $341 \mathrm{LV}$; planche 15, figure 4; Collection : $438 \mathrm{LV}$, PXIII, 56.

Les jeunes schizontes ont un contour irrégulier, sont allongés le long du noyau du GR séparés de lui par un fin espace. Ils contiennent une chromatine granuleuse mal limitée, un cytoplasme peu abondant, et quelques vacuoles blanches. Le pigment est déjà rassemblé sous forme de quelques granules.

En se développant, il s'allonge, reste peu incurvé et dépasse une extrémité du noyau du GR. 12 ou le plus souvent 14 noyaux s'individualisent en bourgeonnant à sa périphérie, en formant deux rangées parallèles de noyaux faisant saillie latéralement. Le cytoplasme central est toujours peu abondant et clair. L'hématie et son noyau ne sont pas modifiés.

Les gamétocytes ne modifient pas la cellule hôte, s'incurvent et s'élargissent au niveau d'un pôle de l'hématie et n'atteignent pas le pôle opposé.

. Diagnostic différentiel

L'espèce doit être comparée à $P$. ghadiriani avec lequel elle coexiste parfois. Elle s'en différencie par sa forme plus droite et plus étroite, par le nombre de noyaux (12-14 au lieu de 18-24), par l'effet sur le GR rarement altéré chez $P$. tranieri, avec son noyau constamment déplacé par $P$. ghadiriani, et enfin et surtout par la disposition des noyaux, périphériques et saillants chez $P$. tranieri, régulièrement dispersés dans le cytoplasme pour $P$. ghadiriani.

$P$. tranieri doit également être comparé aux espèces suivantes :

- P. gundersi Bray, 1962, parasite de Strix woodfordii (Smith, 1834) dont les schizontes et les gamétocytes sont beaucoup plus minces et réguliers que ceux de $P$. tranieri, et dont le cytoplasme ne contient pas de vacuoles, contrairement à ce dernier.

- P. lophurae produit 10-12 noyaux, au lieu de 14 pour $P$. tranieri. De plus, $P$. tranieri a un contour plus régulier et moins amiboïde que $P$. lophurae et, contrairement à ce dernier, ses schizontes n'entourent pas partiellement le noyau du GR.

- P. bigueti produit moins de mérozoïtes que P. tranieri (10-12 au lieu de 12-14). Il s'amenuise vers une extrémité pouvant envoyer un prolongement nucléocytoplasmique dans l'hématie. Il est plus accolé que $P$. tranieri à la membrane du GR en laissant un espace plus large entre le schizonte et le noyau du globule rouge.

Enfin, l'espèce la plus proche morphologiquement est P. fallax selon la description originale et sa redéfinition par Garnham, 1966. Notre espèce en diffère, comme P. ghadiriani ( $c f$. plus haut), par le caractère non amiboïde des jeunes schizontes, l'absence de "filopodia" dans le GR, l'absence de grandes vacuoles blanches. De plus, les gamétocytes de $P$. fallax ont tendance à englober les deux extrémités du noyau du GR, ceux de $P$. tranieri débordent un seul pôle de ce noyau et n'atteignent pas le pôle opposé.

PLASMODIUM LENOBLEI N. SP. (planche 11, figures 1-12)

L'espèce est dédiée à Dominique Richard-Lenoble. Matériel holotype sur la lame : $376 \mathrm{LV}$; planche 15, figure 10; Collection : $438 \mathrm{LV}$, PXIII, 54.

L'espèce est caractérisée par sa position au sommet du GR, coiffant son noyau. Les jeunes schizontes laissent un espace, qui disparaît avec les formes âgées, entre parasite et noyau du GR. Trophozoïtes et schizontes sont larges dans leur partie moyenne, s'amincissant vers les extrémités. Certains ont une extrémité se prolongeant plus bas que l'autre le long du noyau du GR.

Le cytoplasme est abondant, parsemé de vacuoles de différentes tailles. D'abord, grands et mal individualisés, les masses nucléaires se fragmentent en 12 à 14 noyaux, régulièrement dispersés dans le cytoplasme. Le pigment en grains noirs assez grossiers est rassemblé, en position variable, souvent à une extrémité. 
Les gamétocytes sont allongés, minces et arqués, également en position apicale ou sub-apicale. Le GR n'est pas modifié.

\section{- Diagnostic différentiel}

L'espèce doit être comparée à Plasmodium polare Manwell, 1934, redécrit par cet auteur en 1935, parasite de Petrochelidon pyrrhonota (Vieillot, 1817). Celui-ci diffère de notre espèce par son caractère plus trapu, et moins enveloppant pour le noyau du GR et par la présence de travées de chromatine reliant les noyaux des schizontes, non observées chez P. lenoblei. Les gamétocytes de notre espèce sont beaucoup plus minces que ceux de $P$. polare et en position apicale et non latérale.

Nous désignons donc le matériel des Pies françaises sous un autre nom que celui du Petrochelidon américain.

\section{PLASMODIUM DHERTEAE N. SP. \\ (planche 7, figures 1-8)}

L'espèce est dédiée à Michèle Dherte.

Matériel holotype sur la lame : $95 \mathrm{JM}$; planche 15, figure 7; Collection : 438 LV, PXIII, 59.

Schizontes et gamétocytes sont en position latérale, laissant un espace plus ou moins étroit entre parasite et noyau du GR.

Les plus jeunes schizontes identifiés ont 3-4 noyaux assez grands, arrondis, alignés le long du noyau du GR, très peu de cytoplasme et un pigment aggloméré en une masse noire médiane.

Les schizontes, encore immatures, restent allongés parallèlement au noyau du GR, rarement obliques, le dépassant parfois à une extrémité; ils sont de forme générale irrégulière, parfois sinueuse. La répartition des noyaux est irrégulière. Ils sont arrondis ou allongés, d'abord en amas mal limités, séparés par de larges vacuoles, puis disposés en deux rangées irrégulières, bourgeonnant à partir d'un cytoplasme vacuolisé et très peu chromophile ; il existe généralement un noyau terminal. Leur nombre moyen est de huit, les formes à six et dix noyaux n'étant pas rares. Le pigment est aggloméré et latéral.

Schizontes et gamétocytes ne déplacent ni ne modifient l'hématie ou son noyau et, s'ils sont parfois contre ce dernier, il ne semble pas y avoir de réel accolement. Tous deux ont une forme droite, rigide, ou légèrement incurvée. Le pigment du gamétocyte, lorsqu'il est rassemblé, entoure une vacuole.

- Diagnostic différentiel

L'espèce la plus proche est $P$. octamerium Manwell, 1968, parasite de Vidua macroura (Pallas, 1764).

Il s'agit d'un Plasmodium trouvé chez un Vidua importé d'Afrique par un marchand et décrit chez le Canari, Serinus canaria (L., 1758) inoculé avec le sang du Vidua.
Les caractères communs des deux espèces sont le même nombre moyen de noyaux (8), la position latérale dans l'hématie qui n'est pas modifiée et les gamétocytes allongés. Ils diffèrent par l'absence de "filamentous pseudopodium" chez le trophozoïte, la taille du parasite qui peut atteindre presque la longueur du GR parasité par $P$. octamerium et dépasse rarement celle du noyau du GR de $P$. dherteae, enfin, par les gamétocytes larges et encerclant souvent le noyau du GR parasité par $P$. octamerium plus étroits et peu incurvés chez $P$. dherteae.

\section{PLASMODIUM HEXAMERIUM HuFF, 1935}

(planche 12, figure 1-8)

Parasite de Sialia sialis sialis (L., 1758) au Kansas (ÉtatsUnis), décrit chez le Canari inoculé avec le sang du Sialis.

Petit Plasmodium apical ou sub-apical. Les plus jeunes schizontes identifiés à cette espèce ont un cytoplasme bleu, contenant quelques vacuoles blanches et des noyaux périphériques qui, en évoluant, bourgeonnent à la périphérie du cytoplasme. Par la suite, le schizonte devient de forme irrégulière avec le plus souvent six noyaux (exceptionnellement 4 ou 8) qui font saillie vers l'extérieur à partir d'un cytoplasme qui s'éclaircit peu à peu.

Nous n'avons pas observé de différences morphologiques entre notre parasite et la description et les illustrations de cet auteur.

PLASMODIUM VALKIUNASI N. SP.

(planche 10, figures 1-12)

L'espèce est dédiée à Gediminas Valkiûnas.

Matériel holotype sur la lame : $353 \mathrm{LV}$; planche 15, figure 9; Collection : $438 \mathrm{LV}$, PXIII, 60.

Les schizontes ont une forme en éventail et des mérozoïtes allongés très caractéristiques.

La plus jeune forme observée a trois noyaux granuleux, un cytoplasme clair et vacuolé et un pigment rassemblé à l'opposé des noyaux. Elle a déjà une forme caractéristique, triangulaire. Au cours de la multiplication des noyaux, le schizonte acquiert une forme en éventail ; les noyaux sont d'abord arrondis, alignés en arc de cercle disposés le long du côté convexe de l'éventail avec un cytoplasme devenant de plus en plus clair et vacuolé. Le pigment est en grains de petite taille agglomérés lâchement le long du bord plat de l'éventail. Par la suite les noyaux s'allongent formant une palissade en demi-cercle dans laquelle nous avons compté de 12 à 14 noyaux. Leur place dans le GR est apicale ou subapicale, ils l'arrondissent et déplacent son noyau soit vers une extrémité soit latéralement ou enfin, obliquement.

Les gamétocytes sont allongés et, comme les schizontes, arrondissent le GR et déplacent son noyau. Ils ont le bord interne ou les deux bords ondulés et ne sont 


\section{Planche 1 : Plasmodium dorsti}

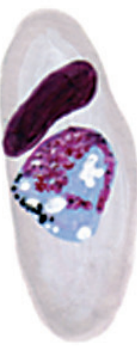

1

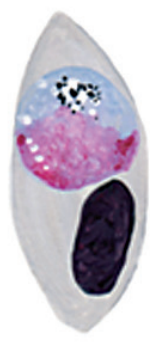

7

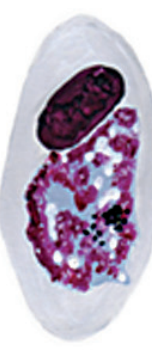

2

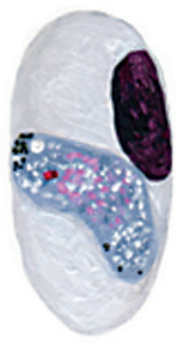

8

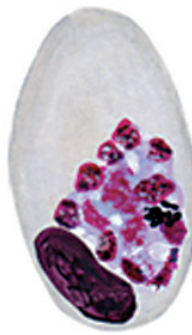

3

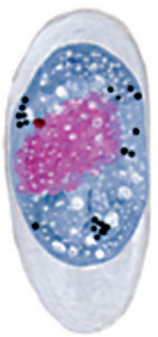

9

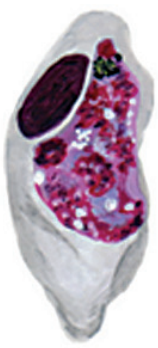

4

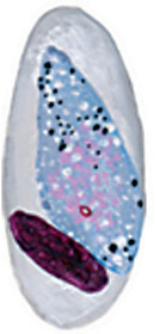

10

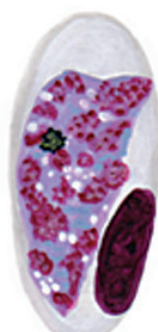

5

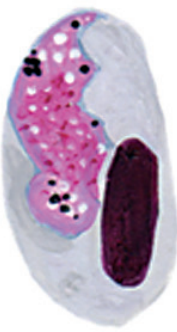

11

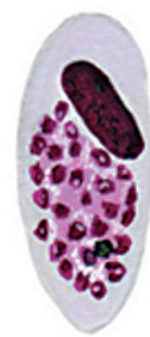

6

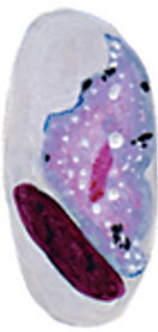

12

\section{Planche 2 : Plasmodium bioccai}

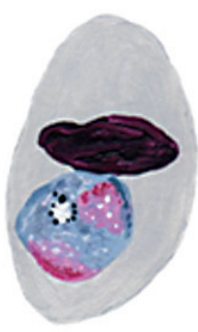

1

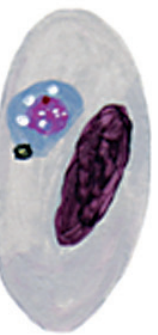

7

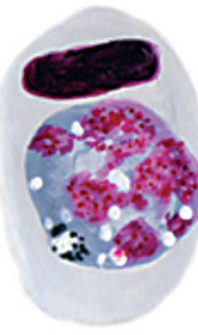

2

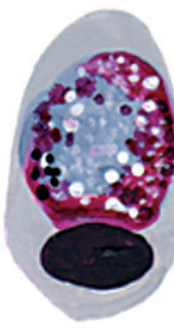

3

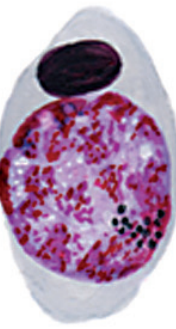

4

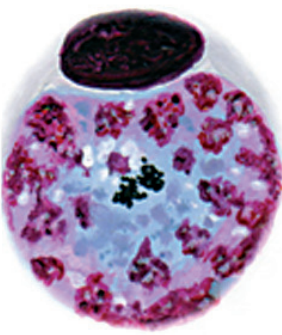

5

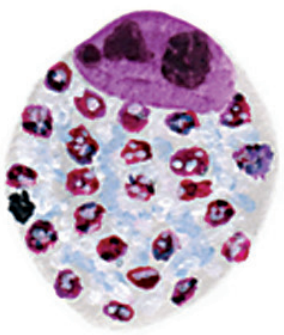

6 


\section{Planche 3 : Plasmodium ghadiriani}
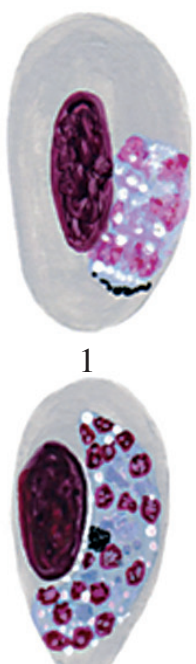

6

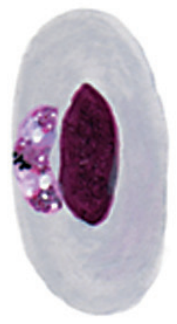

1

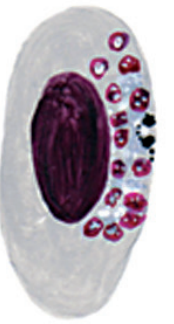

6

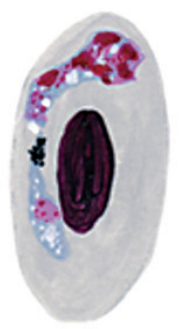

1

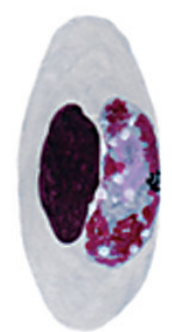

2

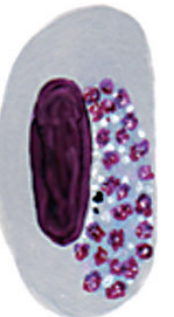

7

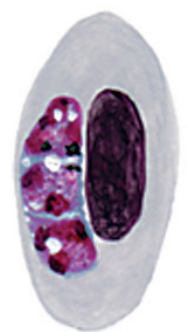

3

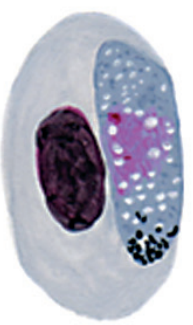

8

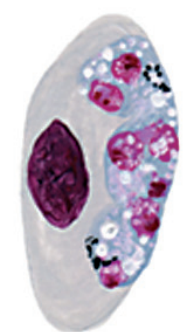

4

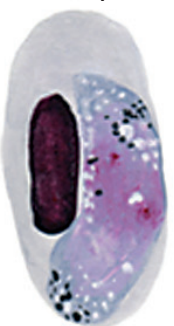

9

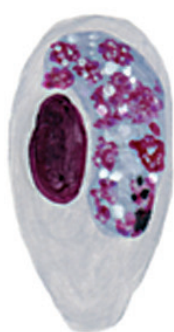

5

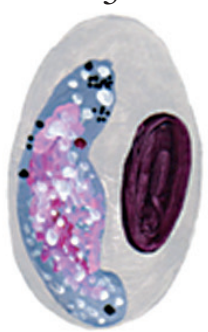

10

\section{Planche 4 : Plasmodium tranieri}

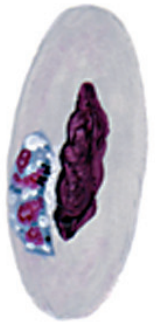

2

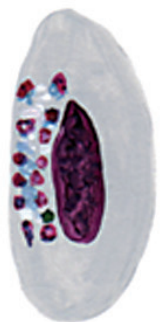

7

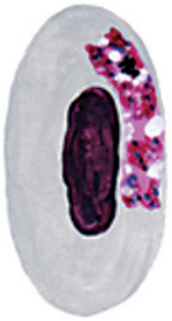

3

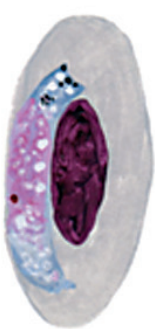

8

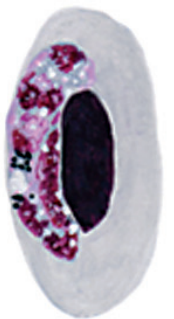

4

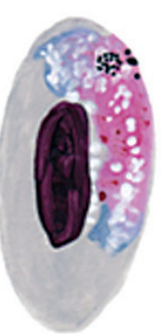

9

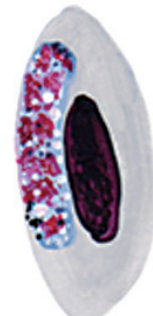

5

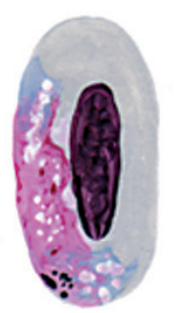

10

\section{Planche 5 : Plasmodium golvani}

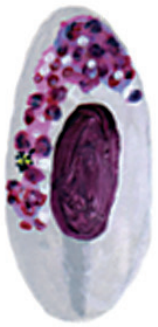

2

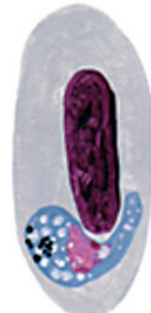

3

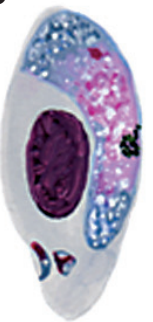

4

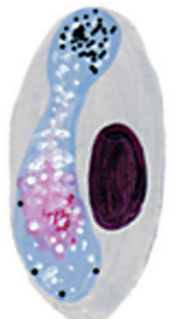

5

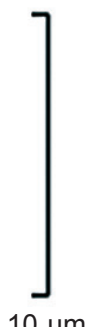

Planche 3. - P. ghadiriani. 1-5: schizontes immatures avec trois masses nucléo-cytoplasmiques; 6-7 : schizontes presque mûrs; 8 : jeune macrogamétocyte; 9 : microgamétocyte mûr; 10 : gamétocyte sénescent.

Planche 4. - P. tranieri. 1-2 : jeunes schizontes; 3-5: schizontes âgés immatures; 6-7 : schizontes presque mûrs; 8 : macrogamétocyte; 910 : microgamétocytes.

Planche 5. - P. golvani. 1 : schizonte jeune; 2 : schizonte presque mûr; 3 : jeune gamétocyte; 4-5: macro- et microgamétocyte. 


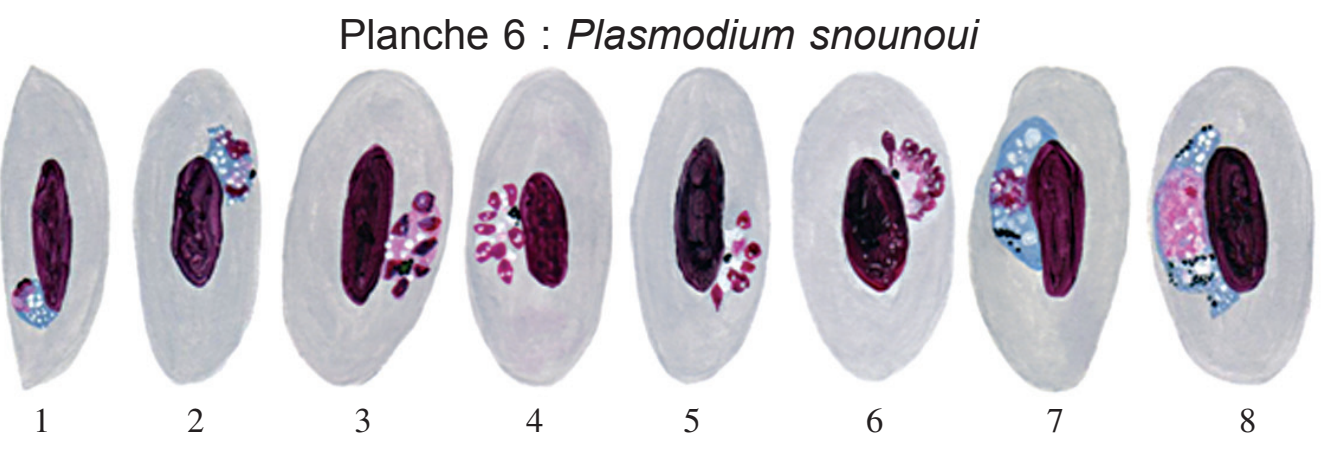

\section{Planche 7 : Plasmodium dherteae}

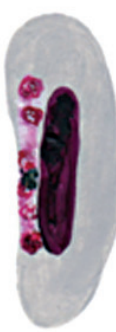

1

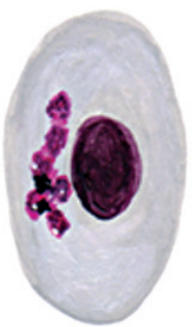

2

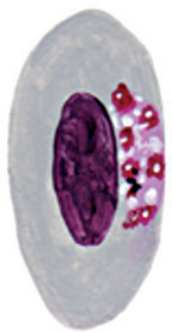

3

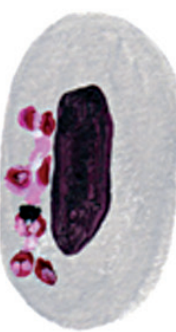

4

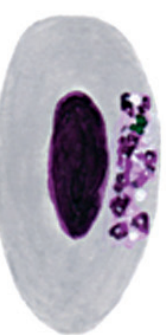

5

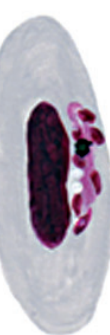

6

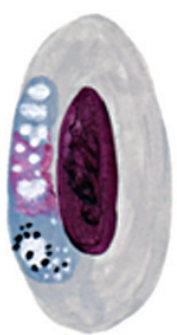

7

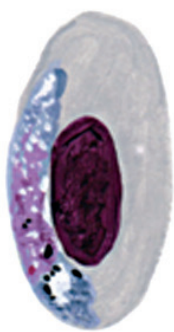

8

\section{Planche 8 : Plasmodium beaucournui}

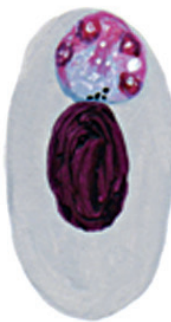

1

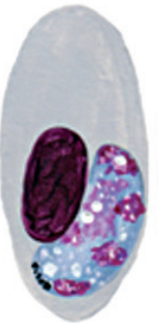

2

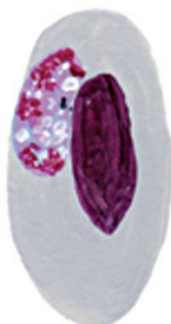

3

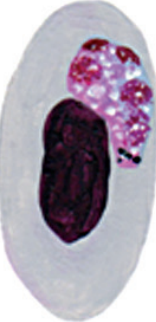

4

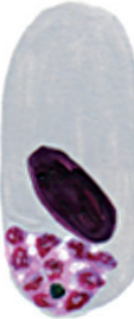

5

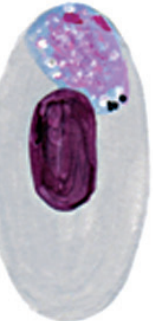

6

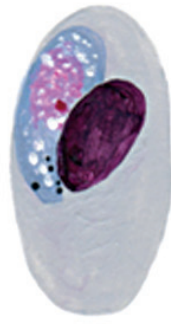

7

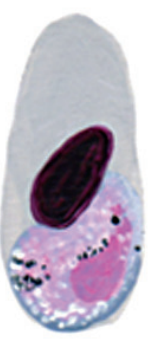

8

Planche 9 : Plasmodium relictum

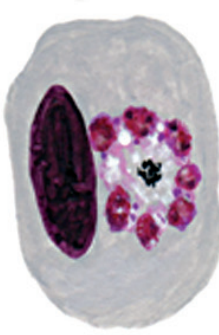

1

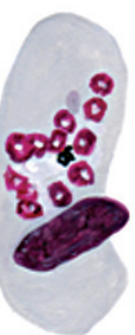

2

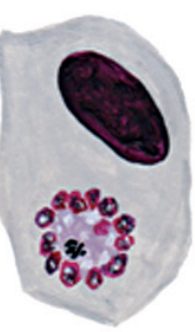

3

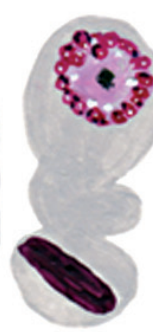

4

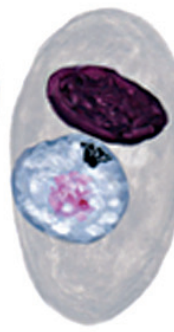

5

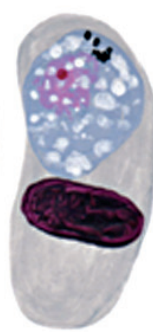

6

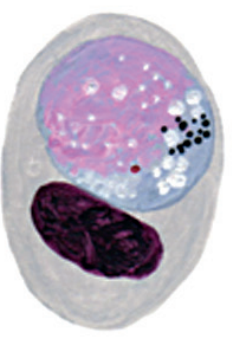

7

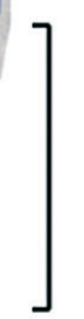

$10 \mu \mathrm{m}$

Planche 6. - P. snounoui. 1 : trophozoïte; 2 : jeune schizonte; 3-6 : schizontes âgés; 7-8 : macro- et microgamétocytes.

Planche 7. - P. dherteae. 1-2 : jeunes schizontes; 3-6 : schizontes âgés; 7-8: macro- et microgamétocytes.

Planche 8. - P. beaucournui. 1-4 : schizontes immatures; 5 : schizonte presque mûr; 6 : jeune gamétocyte; 7 : microgamétocyte; 8 : macrogamétocyte sénescent.

Planche 9. - P. relictum. 1-4 : schizontes; 5 : jeune gamétocyte; 6-7 : macro- et microgamétocytes. 


\section{Planche 10 : Plasmodium valkiunasi}

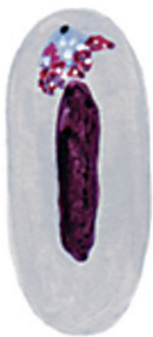

1

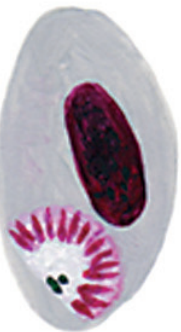

7

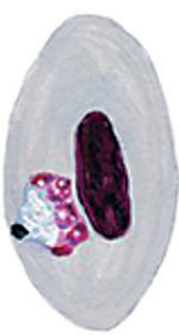

2

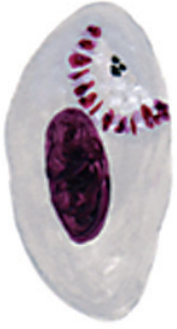

8

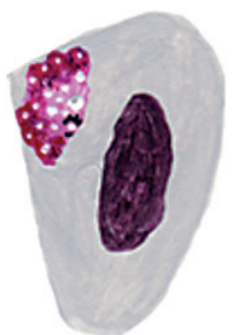

3

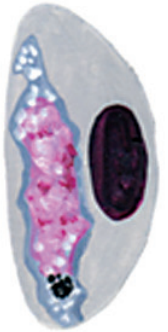

9

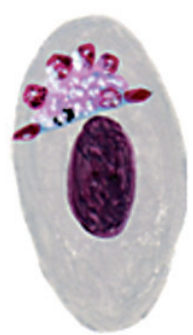

4

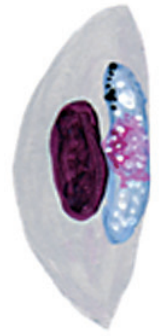

10

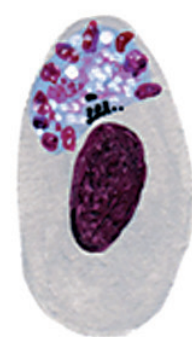

5

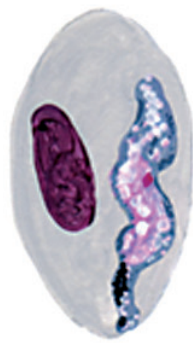

11

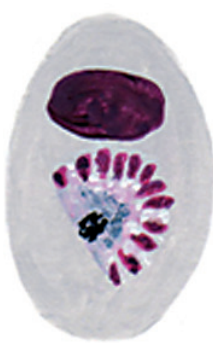

6

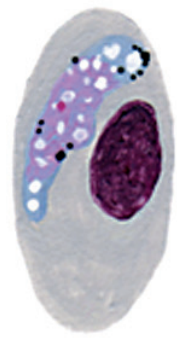

12

Planche 11 : Plasmodium lenoblei

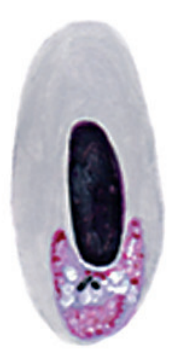

1

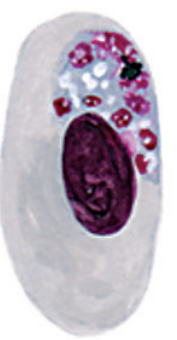

7

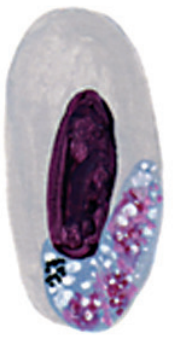

2

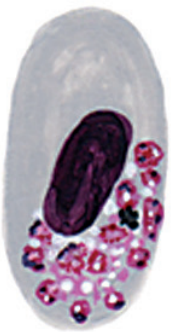

8

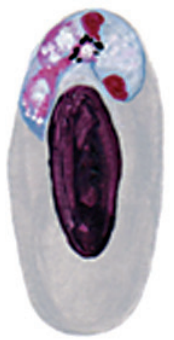

3

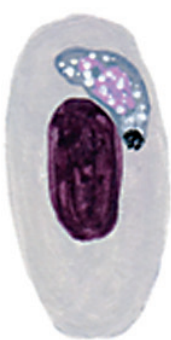

9

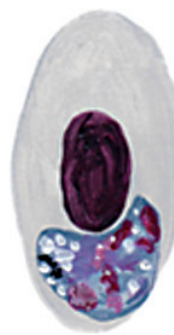

4

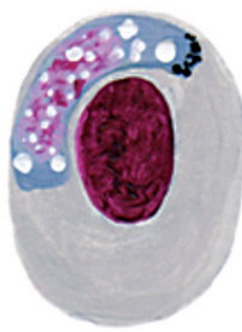

10

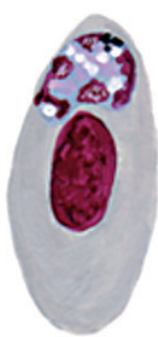

5

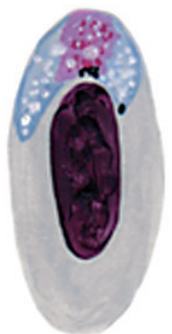

11

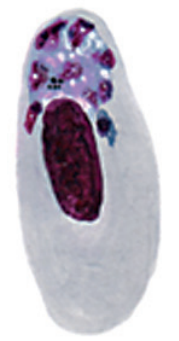

6

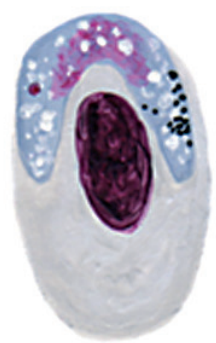

12

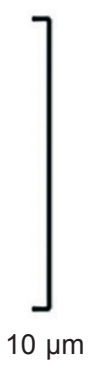

Planche 10. - P. valkiunasi. 1-5: schizontes immatures; 6-8 : schizontes presque mûrs; 9 , 12 : microgamétocytes; 10-11 : macrogamétocytes.

Planche 11. - P. lenoblei. 1-8 : schizontes; 9 : jeune gamétocyte; 10-12 : microgamétocytes; 11 : macrogamétocyte. 
Planche 12 : Plasmodium hexamerium

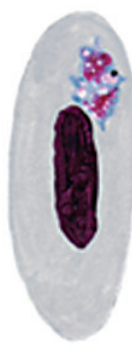

1

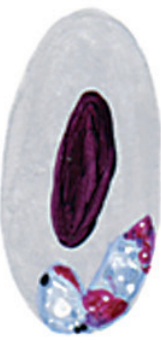

1

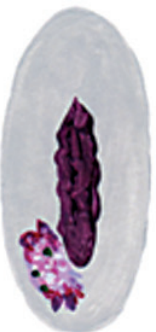

2

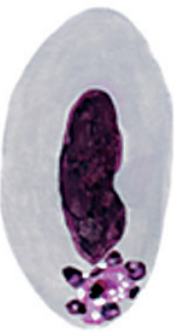

3

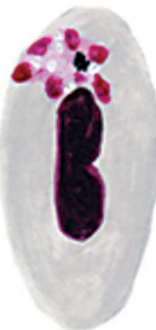

4

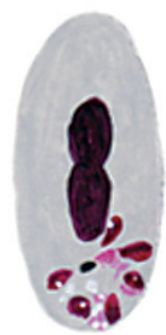

5

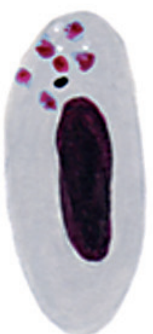

6

Planche 13 : Plasmodium sp. 1

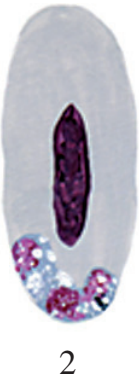

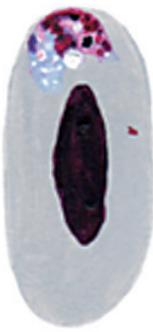

3

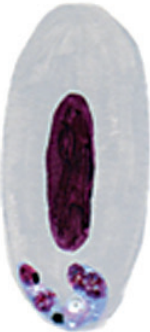

4

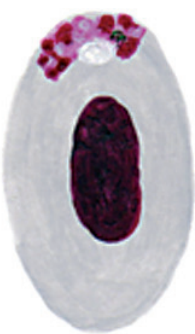

5

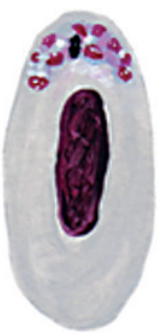

6

Planche 14 : Plasmodium sp. 2 proche P. rouxi

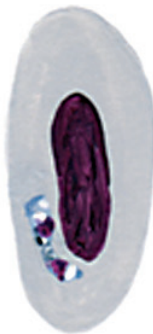

1

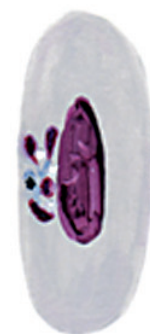

2

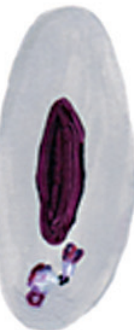

3

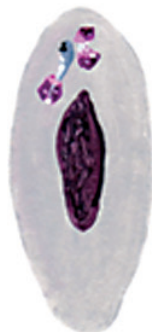

4

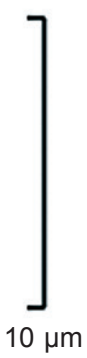

Planche 15 : Photographies des holotypes des nouvelles espèces de Plasmodium décrites

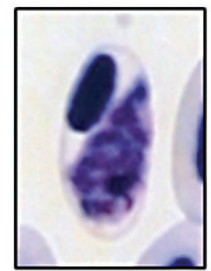

1

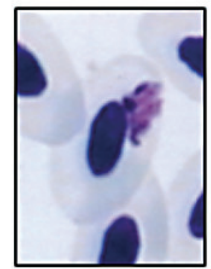

6

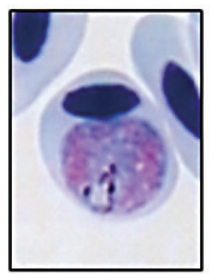

2

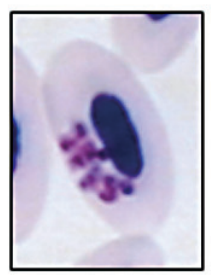

7

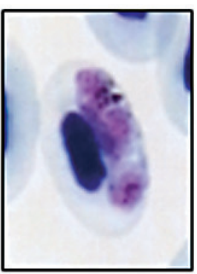

3

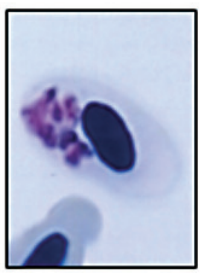

8

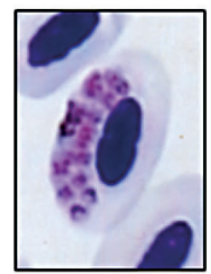

4

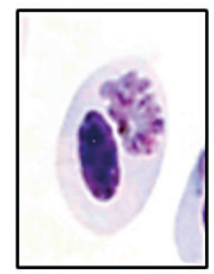

9

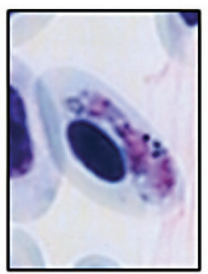

5

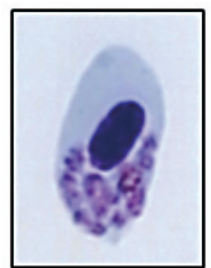

10

Planche 12. $-P$. hexamerium. 1-3 : jeunes schizontes; 4-8 : schizontes presque murs.

Planche 13. $-P$. sp. 1. 1-4 : jeunes schizontes; 5-6 : schizontes âgés.

Planche 14. - P. sp. 2 proche $P$. rouxi. 1-4 : schizontes.

Planche 15. - Photographies des holotypes. 1 : P. dorsti; 2: P. bioccai; 3 : P. ghadiriani ; 4 : P. tranieri; $5:$ P. golvani; $6:$ P. snounoui; 7 : P. dherteae $; 8$ : P. beaucournui $; 9:$ P. valkiunasi $; 10:$ P. lenoblei. 
pas en contact avec le noyau du GR ni avec la membrane de l'hématie.

\section{- Diagnostic différentiel}

Sa forme en éventail ouvert le différencie de tous les Plasmodium décrits chez les oiseaux.

Les mérozoïtes allongés l'apparentent à $P$. elongatum Huff, 1930 décrit à partir d'une souche trouvée chez un Canari captif à Baltimore (États-Unis).

Cependant, aucune forme n'a été observée dans les réticulocytes ce qui est la localisation habituelle de P. elongatum. De plus, la forme triangulaire des schizontes jeunes et en éventail des stades âgés différencie $P$. valkiunasi des formes arrondies jusqu'à maturité, décrites par Huff.

\section{PLASMODIUM SNOUNOUI N. SP. (planche 6, figures 1-8)}

L'espèce est dédiée à Georges Snounou.

Matériel holotype sur la lame : $339 \mathrm{LV}$; planche 15, figure 6; Collection : 438 LV, PXIII, 52.

Les trophozoïtes et les schizontes siègent en position latérale, plus proche d'une extrémité que de l'autre, débordant parfois légèrement un pôle des noyaux du GR auxquels ils sont étroitement accolés. Le cytoplasme est d'abord bleu, puis devient très clair dans le schizonte âgé.

Les noyaux, au nombre de six à huit, sont d'abord arrondis, puis deviennent allongés, bourgeonnant en semi couronne irrégulière à l'opposé du noyau du GR. Le pigment est constitué par un ou deux petits grains chez le trophozoïte ou le schizonte jeune. Il est, chez les schizontes plus âgés, sous forme d'une petite masse noire irrégulière, accolée au noyau du GR.

Nous lui rattachons les gamétocytes allongés présentant la même position dans l'hématie et le caractère "accolé" au noyau du GR.

Ils sont courts, trapus, dépassent très peu le noyau du GR. Au niveau de l'accolement une excroissance du noyau du GR est souvent observée. Un granule chromatinien accessoire peu chromophile est visible.

\section{- Diagnostic différentiel}

L'espèce ne ressemble à aucune autre décrite chez les Oiseaux. Son caractère le plus frappant est son accolement étroit au noyau du GR. Nous le comparons donc à P. nucleophilum Manwell, 1935, parasite de Dumetella carolinensis (L., 1766) qui présente ce caractère. $P$. snounoui diffère de P. nucleophilum par les caractères suivants : forme en semi couronne peu allongée, et non le plus souvent franchement allongée pour P. nucleophilum; nombre de noyaux, huit en moyenne chez le premier, 6-7 chez le second; absence de déplacement du noyau du GR par les schizontes âgés de $P$. snounoui; et position latérale et non apicale, très différente de celle de P. nucleophilum dessiné par Manwell (1935, planche I, figures 15 et 16) qui était considérée par cet auteur comme "especially characteristic".

\section{PLASMODIUM GOLVANI N. SP.} (planche 5, figures 1-5)

L'espèce est dédiée à Yves Golvan.

Matériel holotype sur la lame : $357 \mathrm{LV}$; planche 15, figure 5; Collection : $438 \mathrm{LV}$, PXIII, 53.

L'espèce se développe à la fois au sommet du GR où elle occupe presque tout l'espace et le long du noyau où elle envoie un prolongement, d'abord à prédominance cytoplasmique, puis dans lequel se multiplient également des noyaux.

Nous n'avons observé qu'un seul schizonte proche de la maturité contenant approximativement 28 noyaux. Le pigment est formé par des granules assez fins rassemblés dans le prolongement latéral du schizonte.

Les gamétocytes ont une forme évoquant celle des schizontes, élargis vers l'une des extrémités et amincis le long du noyau du GR. Ce dernier n'est pas modifié par le parasite.

\section{- Diagnostic différentiel}

L'espèce qui nous parait la plus proche de $P$. golvani est $P$. anasum Manwell et Kuntz, 1965, parasite de Anas clypeata L., 1758, qui comme notre parasite occupe le sommet du GR et "s'allonge par une extrémité le long du noyau du GR". Il en diffère par le nombre de noyaux (28 comptés dans un schizonte de $P$. golvani, 18 au maximum pour $P$. anasum). Les gamétocytes de $P$. anasum encerclent le noyau du GR, ceux de $P$. golvani atteignent au maximum les extrémités du GR.

\section{Plasmodium Relictum, (Grassi et Feletti, 1891)}

(planche 9, figures 1-7)

L'espèce observée chez la Pie est très proche de $P$. relictum, parasite de Passer hispaniolensis (Temminck, 1820) de Sicile, mais nous la décrivons néanmoins en raison de quelques faibles différences avec celui-ci.

Le parasite siège le plus souvent dans un GR dont le noyau est déplacé vers une extrémité, partiellement basculé ou perpendiculaire au grand axe. Les schizontes se développant le long du noyau qu'ils repoussent latéralement sont plus rares.

Le GR est fréquemment élargi et très déformable.

Les noyaux sont d'abord assez gros, périphériques peu nombreux puis se divisent, restent plus ou moins périphériques, en couronne, avec un cytoplasme très peu abondant, surtout au centre où une zone claire est observée. Le schizonte a un contour irrégulier, grossièrement arrondi. Les noyaux au nombre de 14 (12 à 18) font légèrement saillie vers l'extérieur.

Le pigment est en grains noirâtres, assez grossiers, rassemblés mais non agglomérés, sauf en fin de maturation. 
Les gamétocytes sont ronds, ellipsoïdes ou ovalaires et ont tendance à s'allonger en vieillissant et à acquérir un contour irrégulier. Ils ont parfois une forme allongée, mais restent relativement courts et déplacent fortement le noyau du globule rouge. Celui-ci a le même caractère déformable qu'avec les autres stades.

- Statut taxonomique

Plasmodium relictum a été décrit par Grassi et Felletti en 1891 chez Passer hispaniolensis en Sicile, puis, redécrit par Raffaele en 1931, chez le même oiseau et dans la même localité. Landau et al., 2003, le redécrivent chez Passer domesticus en France. Le parasite de Pica pica présente quelques faibles différences avec celui du Passer domesticus, en particulier une déformabilité plus prononcée du GR et une couronne de noyaux plus régulière que celle des descriptions précédentes. Ces différences sont très faibles et nous préférons le désigner sous le nom de relictum.

Comme le $P$. relictum du Passer domesticus et du Passer hipaniolensis, le parasite de la Pie se différencie très nettement des deux espèces décrites ci-dessous ( $P$. dorsti n. sp. et P. bioccai n. sp.) et de P. maior Raffaele, 1930, parasite de Passer hipaniolensis par le nombre beaucoup plus faible de noyaux (14 au lieu de 24 à plus de 30) et par leur disposition : ceux de $P$. relictum sont périphériques saillants autour d'un cytoplasme peu abondant, alors qu'ils sont dispersés à l'intérieur de la masse cytoplasmique qui est abondante chez les trois autres.

Corradetti et al., 1970, ont redécrit sommairement les stades érythrocytaires observés chez des Moineaux de la localité d'origine de $P$. relictum en Sicile et déposé des néohapantotypes dans la collection de la Wellcome Foundation. Le nombre moyen de mérozoïtes (14) à maturité correspond bien à celui de $P$. relictum, cependant, le nombre non négligeable de schizontes produisant de 18 à 24 mérozoïtes nous fait penser à une infection concomitante par P. maior.

\section{PLASMODIUM DORSTI N. SP. \\ (planche 1, figures 1-12)}

L'espèce est dédiée à la mémoire de Jean Dorst. Matériel holotype sur la lame : $357 \mathrm{LV}$; planche 15, figure 1; Collection : $438 \mathrm{LV}$, PXIII, 57.

Les schizontes jeunes ont déjà certains des caractères du schizonte âgé : forme irrégulière, cytoplasme abondant et chromatine granuleuse irrégulière, mal limitée. Le noyau du GR est repoussé vers une extrémité, parallèlement au grand axe ou le plus souvent oblique; le GR lui-même est encore modérément arrondi et peu ou pas encore décoloré

Les schizontes âgés ont une forme de plus en plus irrégulière, la chromatine se fragmente en quelques masses granuleuses et de grandes tailles, le GR commence à se décolorer.
Proche de la maturité, le schizonte remplit les 3/4 du GR. Les noyaux des stades précédents se sont fragmentés en une série de plus petites masses de chromatine qui restent d'abord groupées puis se dispersent. Ils peuvent également être disposés en rangées irrégulières, séparées par des zones lacunaires. Le GR est complètement décoloré, à l'exception de la périphérie, il s'élargit et se déforme parfois et son noyau est repoussé contre un bord.

Un schizonte presque mûr a été observé avec 32 noyaux, mais ceux-ci sont, la plupart du temps, impossibles à compter et nous les évaluons à plus de 30.

Le pigment est abondant, noir, d'abord plus ou moins dispersé, puis s'agglutinant pour former, proche de la maturité, une masse noire, réfringente.

Comme les schizontes, les gamétocytes décolorent le GR et déplacent son noyau tantôt latéralement, tantôt vers une extrémité. Ils sont, plus grands que ceux de $P$. bioccai, d'abord ovalaires (formes jeunes) puis allongés ellipsoïdes et enfin déformé, avec des bords irréguliers à fins prolongements.

\section{- Diagnostic différentiel}

Les différences avec $P$. bioccai n. sp. sont détaillées cidessous.

$P$. dorsti diffère de $P$. maior Raffaele, 1930, parasite de Passer, par sa forme beaucoup plus tourmentée, par le nombre de noyaux (28 à plus de 30 pour $P$. dorsti, 20 à 24 pour $P$. maior), par sa plus grande taille, et par les déformations du GR nettement plus importantes qu'avec P. maior.

PLASMODIUM BIOCCAI N. SP.

(planche 2, figures 1-12)

L'espèce est dédiée à la mémoire d'Ettore Biocca. Matériel holotype sur la lame : $344 \mathrm{LV}$; planche 15, figure 2 ; Collection : $438 \mathrm{LV}$, PXIII, 58.

Les schizontes immatures, qu'ils soient jeunes ou plus âgés sont ronds, bien limités, avec un contour régulier.

Le cytoplasme est abondant, avec des noyaux grands, granuleux, mal individualisés, d'abord répartis dans la masse, puis plutôt prédominants en périphérie, mais ne faisant pas saillie vers l'extérieur.

Les schizontes âgés arrondissent complètement le GR, le remplissent presque entièrement et ne le décolorent pas. Le noyau, repoussé contre la membrane, est ellipsoïde ou rond et fréquemment plus petit et dense que les noyaux des GR voisins. Les noyaux des schizontes âgés sont difficiles à compter avant la maturité complète, car mal individualisés. Quelques schizontes mûrs ou presque mûrs contenaient 24 à environ 30 noyaux. Le pigment, noir, grossier, central ou périphérique, est souvent associé à une vacuole.

Les gamétocytes sont ronds ou presque, avec un granule chromatinien accessoire net et un pigment réparti en fonction de leur âge comme illustré (figures 2, 7-12). 
- Diagnostic différentiel

$P$. bioccai diffère de $P$. dorsti par la forme générale régulière chez le premier, irrégulière chez le second. $P$. bioccai diffère également de $P$. dorsti par le nombre de noyaux à maturité, un peu moins élevé (24 à 30 pour le premier contre 28 à plus de 30 pour le second) et par l'effet sur le GR : arrondi, non décoloré avec un noyau dense, repoussé vers un pôle du GR perpendiculairement à son grand axe par $P$. bioccai, élargi et surtout décoloré, avec un noyau allongé et basculé obliquement ou allongé contre la membrane du GR par $P$. dorsti. Les noyaux des formes âgées sont prédominants à la périphérie chez $P$. bioccai, répartis dans la masse du schizonte chez $P$. dorsti.

$P$. bioccai et $P$. maior ont en commun l'abondance du cytoplasme, mais le premier est plus grand et a un nombre supérieur de noyaux (24 à 30 vs 20 à 24). P. maior a un contour irrégulier alors que celui de $P$. bioccai est lisse.

\section{PLASMODIUM BEAUCOURNUI N. SP. (planche 8, figures 1-8)}

L'espèce est dédiée à Jean-Claude Beaucournu. Matériel holotype sur la lame : $339 \mathrm{LV}$; planche 15, figure 8; Collection : $438 \mathrm{LV}$, PXIII, 51.

Les jeunes schizontes à 4-6 noyaux siègent au sommet du GR, ou en position latéro-apicale, le plus souvent déjà en contact avec le noyau du GR. Les noyaux sont périphériques, granuleux, grands, mal limités, le cytoplasme abondant parsemé de vacuoles. Par la suite, les schizontes s'allongent, pouvant atteindre les deux tiers de la longueur du noyau du GR et occupent toute la largeur de l'hématie tout en s'incurvant au contact du noyau. Le pigment est d'abord formé par des grains individuels noirs rassemblés sur une petite surface et s'agglomère à maturité en une à deux masses réfringentes.

Le schizonte mûr a un contour irrégulier, quelques noyaux faisant saillie vers l'extérieur, et produit 12 à 14 noyaux.

Le noyau du GR est souvent légèrement basculé, comme repoussé par le parasite, mais la cellule hôte ne présente pas d'autres anomalies.

Les gamétocytes présentent les mêmes caractères (forme, position, effet sur le GR) que les schizontes. Ils sont apicaux ou latéro-apicaux. Leur forme ellipsoïde, plus ou moins allongée, nous incite à les rattacher des gamétocytes de type rond.

- Diagnostic différentiel

P. hegneri Manwel et Kuntz, 1966, parasite de Anas crecca crecca L. 1758, nous paraît être l'espèce la plus proche de la nôtre. Cependant, les auteurs ont probablement eu affaire à un mélange d'espèces. Certains stades décrits sous le nom de $P$. hegneri sont proches des stades équivalents de $P$. beaucournui : trophozoïtes accolés au noyau du GR, schizontes apicaux ou sub- apicaux, évoluant en contact du noyau du GR, gamétocytes ronds ou ovales.

Les deux espèces diffèrent par, l'absence dans le trophozoïte de $P$. beaucournui de grandes vacuoles blanches et de fins pseudopodes chez le jeune schizonte, caractéristiques de $P$. hegneri.

PLASMODIUM SP. 1

(planche 13, figures 1-6)

Les parasites siègent au sommet du GR, contre la membrane apicale, laissant un large espace entre eux et le noyau du GR; ils sont allongés, incurvés, épousant la forme du GR. Le jeune trophozoïte est déjà allongé et contient une grande vacuole. Les plus jeunes schizontes observés ont presque la taille des schizontes mûrs, mais ne contiennent que deux noyaux espacés et quelques grandes vacuoles.

Les schizontes proches de la maturité contiennent huit à dix noyaux de petite taille et une grande vacuole blanche ou plusieurs plus petites. Le pigment est rassemblé en un amas noir médian ou submédian.

- Diagnostic différentiel

En raison de sa localisation au sommet du GR et de sa forme, Plasmodium sp. 1 doit être comparé à P. lenoblei. Il en diffère par sa plus petite taille, le nombre de noyaux, huit à dix contre 12 à 14 pour $P$. lenoblei, par son éloignement du noyau du GR qui est coiffé par $P$. lenoblei et par la plus grande taille des vacuoles.

\section{PLASMODIUM SP. 2 PROCHE P. ROUXI \\ (planche 14, figures 1-4)}

C'est un petit parasite dont les schizontes ont quatre noyaux disposés par paires opposées, rattachées au centre par un fin filament de cytoplasme. Le pigment est représenté par trois grains centraux. Nous n'avons trouvé que trois schizontes de ce type et pas de gamétocytes.

\section{- Diagnostic différentiel}

Il est proche de P. rouxi Sergent et al., 1928, parasite de Passer hispaniolensis en Algérie; les formes observées n'en diffèrent que par la quantité de pigment produite (trois grains au lieu de deux). Étant donné le faible nombre de formes trouvées nous ne pouvons pas le différencier du parasite du Moineau algérien.

\section{Classification des PlasmoDium identifiés}

Le tableau II indique la liste des Plasmodium identifiés chez chacune des Pies et le tableau I est une classification dichotomique de ces Plasmodium. Cependant, ces données sont incomplètes puisque les formes jeunes, en particulier celles des petites espèces, ne peuvent être identifiées.

D'après le tableau II, les espèces $P$. bioccai (très proche de $P$. maior) et $P$. relictum, étant donné leur grande fréquence, pourraient constituer des formes ancestrales. 
1-(8) gamétocytes arrondis ou ovalaires.

2-(3) parasite adossé au noyau du GR qui est peu déplacé, GR non déformé ou décoloré, position apico-latérale .......... beaucournui

3-(2) parasite non adossé au noyau du GR qui est fortement déplacé, GR déformé ou décoloré, position médiane.

4-(5) GR agrandi et déformé irrégulièrement, schizontes en rosace, noyaux du schizonte périphériques, saillants, cytoplasme central, clair, peu abondant, 14 noyaux .

5-(4) GR agrandi et arrondi régulièrement, noyaux du schizonte répartis dans le cytoplasme, cytoplasme abondant, 24 à plus de 30 noyaux.

6-(7) GR non éclairci, schizonte arrondi à un contour lisse, pigment en rapport avec une vacuole, 24 à 30

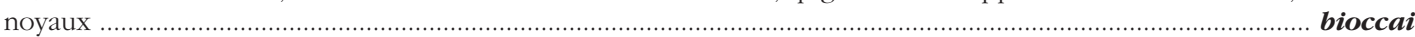
7-(6) GR décoloré, schizonte de forme irrégulière, pigment sans vacuole associée, plus de 30 noyaux ................. dorsti 8-(1) gamétocytes allongés.

9-(10) schizontes et gamétocytes adhérents au noyau du GR, cytoplasme peu abondant, noyaux groupés du côté opposé au noyau du GR

10-(9) schizontes et gamétocytes non adhérents au noyau du GR.

11-(12) schizontes en éventail, GR déformé noyaux des schizontes allongés, 14 noyaux.

snounoui

valkiunasi 12-(11) schizontes non en éventail, GR non déformé, noyaux non allongés.

13-(14) petite espèce n'ayant que 4 noyaux 14-(13) espèces avec au moins 6 noyaux

15-(16) schizontes d'abord apicaux et envoyant une digitation le long du noyau du GR, 28 noyaux ......... golvani 16-(15) schizonte latéral ou apical, n'émettant pas de longue digitation le long du noyau du GR. 17-(22) schizontes apicaux.

18-(19) schizontes grossièrement arrondis, 6 noyaux

19-(18) schizonte allongé, plus de 6 noyaux.

20-(21) Schizonte éloigné du noyau du GR, 8-10 noyaux, grande vacuole ............. sp. 1

21-(20) Schizonte coiffant le noyau du GR, 12-14 noyaux, cytoplasme avec petites vacuoles

22-(17) schizontes latéraux.

23-(26) schizontes grands ou moyens, à bords parallèles, noyaux régulièrement distribués. 24-(25) schizonte grand, cytoplasme abondant, noyaux répartis dans la masse du schizonte, repousse le noyau du GR, 18-24 noyaux

ghadiriani 25-(24) schizonte moyen, cytoplasme peu abondant, ne repousse pas le noyau du GR, 12-14 noyaux ............................................................. tranieri

26-(23) schizonte petit, à bords non parallèles, noyaux irrégulièrement distribués,

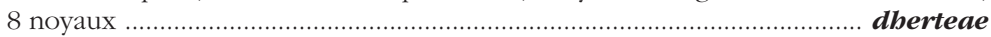

Tableau I. - Tableau dichotomique des espèces identifiées chez Pica pica d'Ile-de-France.

De plus, les groupements d'espèces mis en évidence dans le tableau dichotomique (tableau I) : P. relictum, $P$. bioccai, P. dorsti d'une part et P. tranieri, P. ghadiriani, $P$. dherteae d'autre part se retrouvent également, souvent dans le même hôte.

Connaissant la rapidité des phénomènes de spéciation (Chabaud et Durette-Desset, 1978), l'analyse croisée des deux tableaux pourrait laisser supposer des spéciations successives récentes. Mais, les données fournies par le tableau II, sont trop partielles pour étayer ces hypothèses.

Les données du tableau III mettent en évidence un nombre d'espèces plus important au parc de la Courneuve (14) qu'au parc du Sausset (7). Le parc de la Courneuve avec une superficie deux fois supérieures (400 ha) à celle du parc du Sausset (200 ha), abrite une population estimée à 715 individus (avec un intervalle de confiance : [571-927]), alors qu'au parc du Sausset, la population est estimée à 170 individus (avec un intervalle de confiance : [119-274]) (Chinon et Julliard, 2007). On constate que la densité moyenne en Pies est supérieure au parc de la Courneuve ( 2 individus/ha), par rapport au Sausset (1,25 individus/ha), ce qui pourrait peut-être expliquer les différences dans le nombre espèces de Plasmodium.

\section{DISCUSSION ET CONCLUSIONS}

\section{Systématique des Plasmodium aViaires}

T es caractères qui distinguent les Plasmodium d'Oiseaux sont souvent subtils et la solution généralement adoptée consiste à donner un seul nom, en supposant, lorsque la morphologie d'un parasite n'est pas identique, qu'elle a changé en passant d'un animal à l'autre. Il nous paraît donc dangereux, dans ce cas, de proposer des synonymies surtout lorsque l'hôte et la région géographique sont différents.

Une mise en synonymie éventuelle peut toujours être faite à la suite d'un travail expérimental mais ne peut être admise lorsqu'elle est faite par simple intuition.

\section{SpéCiations MUlTiPles DeS PLASMODIUM}

De nombreux exemples indiquent que la multiplication des espèces à l'intérieur d'un seul et même genre est un phénomène très courant en parasitologie. Dans nombre de cas, la morphologie d'un stade particulier (adulte chez les helminthes, oocyste chez les coccidies), permet une différenciation morphologique relativement facile. Elle met en évidence un polyparasitisme 
CHAVATTE J.M., CHIRON F., CHABAUD A. \& LANDAU I.

\begin{tabular}{|c|c|c|c|c|c|}
\hline \multirow{2}{*}{$\begin{array}{c}\begin{array}{c}\text { Numéro } \\
\text { de Pies }\end{array} \\
1\end{array}$} & \multirow{2}{*}{$\begin{array}{c}\text { Date de capture } \\
11 / 04 / 2005\end{array}$} & \multirow{2}{*}{$\begin{array}{c}\text { Parc de capture } \\
\text { Courneuve }\end{array}$} & \multicolumn{2}{|c|}{ Espèces de Plasmodium } & \multirow{2}{*}{$\begin{array}{c}\text { Autres hématozoaires } \\
\text { Haemoproteus }\end{array}$} \\
\hline & & & $\begin{array}{l}\text { P. beaucournui } \\
\text { P. dherteae } \\
\text { P. hexamerium } \\
\text { P. snounoui }\end{array}$ & $\begin{array}{l}\text { P. valkiunasi } \\
P . \text { sp. } 1 \\
P . \text { sp. } 2\end{array}$ & \\
\hline 2 & $11 / 04 / 2005$ & Courneuve & P. relictum & P. tranieri & Haemoproteus \\
\hline 3 & $11 / 04 / 2005$ & Courneuve & $\begin{array}{l}\text { P. dherteae } \\
\text { P. hexamerium }\end{array}$ & P. sp. 2 & $\begin{array}{l}\text { Haemoproteus } \\
\text { Leucocytozoon }\end{array}$ \\
\hline 4 & $12 / 04 / 2005$ & Courneuve & $\begin{array}{l}\text { P. bioccai } \\
\text { P. dherteae } \\
\text { P. ghadiriani }\end{array}$ & $\begin{array}{l}\text { P. relictum } \\
P . \text { snounoui } \\
\text { P. tranieri }\end{array}$ & Haemoproteus \\
\hline 5 & $12 / 04 / 2005$ & Courneuve & $\begin{array}{l}\text { P. dherteae } \\
\text { P. ghadiriani } \\
\text { P. hexamerium }\end{array}$ & $\begin{array}{l}\text { P. lenoblei } \\
\text { P. relictum } \\
\text { P. tranieri }\end{array}$ & $\begin{array}{l}\text { Haemoproteus } \\
\text { Trypanosoma }\end{array}$ \\
\hline 6 & $12 / 4 / 2005$ & Courneuve & $\begin{array}{l}\text { P. bioccai } \\
\text { P.dorsti }\end{array}$ & P. relictum & Trypanosoma \\
\hline 7 & $12 / 04 / 2005$ & Courneuve & $\begin{array}{l}\text { P. bioccai } \\
\text { P. dorsti }\end{array}$ & P. relictum & \\
\hline 8 & $04 / 05 / 2005$ & Courneuve & P. hexamerium & P. relictum & Haemoproteus \\
\hline 9 & $04 / 05 / 2005$ & Courneuve & $\begin{array}{l}\text { P. dherteae } \\
\text { P. golvani } \\
\text { P. relictum }\end{array}$ & $\begin{array}{l}\text { P. snounoui } \\
\text { P. tranieri } \\
\text { P. valkiunasi }\end{array}$ & Haemoproteus \\
\hline 10 & $19 / 05 / 2005$ & Courneuve & P. bioccai & & Haemoproteus \\
\hline 11 & $19 / 05 / 2005$ & Courneuve & & & Haemoproteus \\
\hline 12 & $31 / 05 / 2005$ & Courneuve & & & Haemoproteus \\
\hline 13 & $02 / 06 / 2005$ & Courneuve & P. bioccai & P. relictum & Haemoproteus \\
\hline 14 & $07 / 06 / 2005$ & Courneuve & P. dherteae & P. ghadiriani & Haemoproteus \\
\hline 15 & $21 / 06 / 2005$ & Courneuve & $\begin{array}{l}\text { P. bioccai } \\
\text { P. dorsti }\end{array}$ & P. tranieri & Haemoproteus \\
\hline 16 & $07 / 04 / 2005$ & Sausset & P. ghadiriani & & Haemoproteus \\
\hline 17 & $07 / 04 / 205$ & Sausset & P. bioccai & & \\
\hline 18 & $04 / 05 / 2005$ & Sausset & $\begin{array}{l}\text { P. bioccai } \\
\text { P. dherteae } \\
\text { P. dorsti }\end{array}$ & $\begin{array}{l}\text { P. gabdiriani } \\
\text { P. relictum } \\
\text { P. tranieri }\end{array}$ & \\
\hline 19 & $07 / 04 / 2005$ & Sausset & P. tranieri & & $\begin{array}{l}\text { Haemoproteus } \\
\text { Lank esterella }\end{array}$ \\
\hline 20 & $13 / 04 / 2005$ & Sausset & $\begin{array}{l}\text { P. bioccai } \\
\text { P. dherteae }\end{array}$ & P. ghadiriani & \\
\hline 21 & $13 / 04 / 2005$ & Sausset & P. tranieri & & \\
\hline 22 & $13 / 04 / 2005$ & Sausset & P. bioccai & P. relictum & \\
\hline 23 & $02 / 05 / 2005$ & Sausset & P. bioccai & & \\
\hline 24 & $01 / 06 / 2005$ & Sausset & P. bioccai & P. tranieri & \\
\hline 25 & $01 / 06 / 2005$ & Sausset & P. dorsti & & \\
\hline 26 & $01 / 06 / 2005$ & Sausset & P. bioccai & P. dorsti & Haemoproteus \\
\hline 27 & $27 / 04 / 2006$ & Sausset & $\begin{array}{l}\text { P. dorsti } \\
\text { P. relictum }\end{array}$ & P. tranieri & \\
\hline 28 & $27 / 04 / 2006$ & Sausset & P. bioccai & & \\
\hline 29 & $27 / 04 / 2006$ & Sausset & & & Haemoproteus \\
\hline 30 & $27 / 04 / 2006$ & Sausset & P. ghadiriani & & Haemoproteus \\
\hline 31 & $27 / 04 / 2006$ & Sausset & & & Haemoproteus \\
\hline 32 & $27 / 04 / 2006$ & Sausset & $\begin{array}{l}\text { P. bioccai } \\
\text { P. dherteae } \\
\text { P. dorsti }\end{array}$ & $\begin{array}{l}P . \text { relictum } \\
P . \text { snounoui }\end{array}$ & Lankesterella \\
\hline 33 & $27 / 04 / 2006$ & Sausset & & & Haemoproteus \\
\hline
\end{tabular}

Tableau II. - Tableau des espèces de Plasmodium et autres hématozoaires détectés chez les Pies des deux parcs. 


\begin{tabular}{lrrr}
\hline & $\begin{array}{c}\text { Pour les } \\
\text { 2 parcs }\end{array}$ & $\begin{array}{c}\text { Cour- } \\
\text { neuve }\end{array}$ & Sausset \\
\hline Total Pies & $\mathbf{3 3}$ & $\mathbf{1 5}$ & $\mathbf{1 8}$ \\
P. bioccai & 15 & 6 & 9 \\
P. relictum & 12 & 8 & 4 \\
P. tranieri & 10 & 5 & 5 \\
P. dherteae & 9 & 6 & 3 \\
P. dorsti & 8 & 3 & 5 \\
P. ghadiriani & 7 & 3 & 4 \\
P. hexamerium & 4 & 4 & 0 \\
P. snounoui & 4 & 3 & 1 \\
P. valkiunasi & 2 & 2 & 0 \\
P. sp. 2 proche P. rouxi & 2 & 2 & 0 \\
P. beaucournui & 1 & 1 & 0 \\
P. golvani & 1 & 1 & 0 \\
P. lenoblei & 1 & 1 & 0 \\
P. sp. 1 & 1 & 1 & 0 \\
Total espèces de Plasmodium & $\mathbf{1 4}$ & $\mathbf{1 4}$ & 7 \\
Total Pies positives pour Plasmodium & $\mathbf{2 8}$ & $\mathbf{1 3}$ & $\mathbf{1 5}$ \\
Total Pies positives pour Haemoproteus & 21 & 13 & 8 \\
Total Pies positives pour Leucocytozoon & 1 & 1 & 0 \\
Total Pies positives pour Trypanosoma & 2 & 2 & 0 \\
Total pies positives pour Lankesterella & 2 & 0 & 2 \\
\hline
\end{tabular}

Tableau III. - Fréquence des différentes espèces de Plasmodium et des autres hématozoaires chez les Pies des deux parcs.

indiscutable. Dans le cas des Plasmodium, l'analyse purement morphologique est plus délicate puisqu'il n'y a pas de structure durable.

Les analyses moléculaires qui seraient importantes pour préciser ces spéciations multiples ne peuvent être que secondaires à l'analyse morphologique en raison du polyparasitisme presque constant des infections naturelles.

\section{MOdes D'ISOLEMENTS ET DE SPÉCIATIONS CHEZ les PlasmodiUM DE PIES PÉRIURBaines}

Les biologistes admettent tous que le phénomène de spéciation est étroitement lié à l'isolement. En dehors des espèces fossiles qui vivent dans un milieu parfaitement stable tout isolement va déterminer une spéciation.

Les Plasmodium de la Pie nous font soupçonner l'existence d'un nouveau mécanisme de spéciation que nous désignons comme "isolement par fidélisation du couple hôte-vecteur".

Nous pouvons donc considérer quatre modalités de spéciation :

1 - Isolement géographique qui ne présente pas de particularités chez les parasites.

2 - Isolement par niches trophiques. La parasitologie nous offre des exemples très clairs de cette spéciation par colonisation d'organes ou de cellules différentes : les Oxyures de tortue (Petter, 1966), les Coccidies du Moineau (Grulet et al., 1982), les Trichostrongylides (Chabaud et Durette-Desset, 1978) occupent différents niveaux du tube digestif ou à un même niveau des zones différentes de la villosité.
Le phénomène est plus discret dans le cas des Plasmodium de la Pie, mais, comme nos descriptions le soulignent, les différentes espèces ont des localisations précises à l'intérieur de l'hématie.

3 - Niches horaires : l'exemple le plus démonstratif est celui des Isospora du Moineau (Grulet et al., 1982), dont les fèces sont négatives pendant la matinée, deviennent positives l'après midi, chaque espèce étant excrétée pendant une tranche horaire différente. Ce facteur ne peut être négligé chez les Plasmodium qui ont un cycle nycthéméral bien défini.

4 - Isolement par fidélisation du couple hôte-vecteur.

\section{FidÉLISATION DU COUPLE HÔTE-VECTEUR}

La diversité spécifique des Plasmodium parasites de la Pie est inattendue. Son explication réside à notre avis dans la biologie de l'hôte et du vecteur.

1 - Éthologie de la Pie : l'espèce est temporairement grégaire en hiver (hors de la période de transmission), période pendant laquelle de vastes rassemblements (dortoirs) sont observés (Birkhead, 1991). Dès le mois de mars, les couples formés au sein des parcs urbains sont cantonnés à leur territoire pour toute la période de reproduction (jusque fin juillet), et s'opposent le plus souvent à l'intrusion d'autres Pies. Les partenaires de chaque couple sont fidèles d'une année sur l'autre, de même qu'à leur territoire. L'appariement et le comportement territorial de la Pie pourraient impliquer un véritable isolement des couples même si les sites d'étude abritent de fortes densités de couples nicheurs. La fidélité au territoire et son occupation par le couple presque toute l'année seraient plus marquées parmi les Pies nichant dans les habitats urbains (Chiron, com. pers.). De plus, les populations présentes dans les habitats urbains et périurbains sont de plus en plus denses alors que la Pie disparaît des habitats peu urbanisés (Chiron et al., 2006, soumis), ce qui favoriserait ce phénomène.

2 - Biologie des Aedes : nous incriminons les Aedes comme étant les principaux vecteurs de ces parasites. En effet, les infections des Pies sont très précoces, surtout au cours de l'année 2005 (puisque toutes les Pies sont positives dès le 11 avril) et apparaissent plus tôt qu'en 2006 dont l'hiver a été prolongé (trois Pies négatives sur sept le 27 avril). Cette précocité de l'infection nous fait donc penser que ce sont les moustiques du genre Aedes qui sont les vecteurs naturels. En effet, même si quelques rares spécimens de Culex et Culiseta hibernent, ce sont les Aedes qui éclosent dès les premières pluies printanières et émergent ensuite en masse dès les premières chaleurs. Le Genre Aedes est très représenté en France, puisqu'il regroupe 27 des 63 espèces des Culicidæ de la faune française (Schaffner, 1998) et qu'en région Île-de-France, on en compte environ 14 espèces (Rageau et al., 1970) qui présen- 
tent chacune des particularités éthologiques propres. Les gites larvaires des Aedes, constitués par des collections d'eaux temporaires sont beaucoup plus variés que ceux des autres Culicides et sont constitués dès la fin de l'hiver. Les relations Pies-vecteurs sont donc très variables d'un gîte à l'autre tout en étant stables d'une année à l'autre.

Il existe donc une multitude de micro-foyers où s'est établie, au cours des temps, une véritable fidélisation entre l'hôte et le vecteur, donc un isolement, et au cours de l'évolution une spéciation.

\section{REMERCIEMENTS}

$\mathrm{N}$ ous remercions le Ministère de l'agriculture, de l'alimentation, de la pêche et de la ruralité, et le Ministère de l'environnement pour nous avoir autorisé la capture, le transport et la détention des Pies de l'étude. Nous sommes reconnaissants à Denis Couvet et à la Direction du jardin des plantes qui nous a accordé toutes les facilités nécessaires à l'examen des Oiseaux. Nous remercions sincèrement A. Boucher de l'Office national de la chasse et de la faune sauvage pour ses conseils et son aide. Nous remercions également F. Gonnet-Gonzalez et S. Bertani pour leur aide durant ce travail.

\section{RÉFÉRENCES}

BiRkHEAD T. The magpies. London: T \& AD Poyser Ltd., 1991, pp. 270 .

Bray R.S. On the parasitic Protozoa of Liberia. VII - Haemosporidia of the owls. Archives de l'Institut Pasteur Algérie, 1962, 40, 201-207.

BRUMPT E. Paludisme aviaire : Plasmodium gallinaceum n. sp. de la Poule domestique. Comptes Rendus Hebdomadaires des Séances de l'Académie des Sciences, Paris, 1935, 200, 783-785.

Chabaud A.G. \& Durette-Desset M.C. Parasitisme par plusieurs espèces congénériques. Bulletin de la Société Zoologique de France, 1978, 103 (4), 459-464.

Chiron F. \& Julliard R. Responses of songbirds to Black-billed magpie regulation in urban habitat. Journal of Wildlife Management (submitted).

Chiron F., Lee A. \& Julliard R. Magpie dynamic in a dynamic landscape: new insight from an innovative methodology. Oikos (submitted).

Coggeshall L.T. Plasmodium lophurae, a new species of malaria parasite pathogenic for the Domestic Fowl. American Journal of Hygiene, 1938, 27, 615-618.

Corradetti A., Garnham P.C.C., Neri I., Scanga M. \& CavalLINI C. A redescription of Plasmodium (Haemaboeba) relictum (Grassi and Feletti, 1891). Parassitologia, 1970, 12, $1-10$.
GARnham P.C.C. Malaria parasites and other Haemosporidia. Oxford: Blackwell, 1966, pp. 1114.

Grassi B. \& Feletti R. Malariaparasiten in den Vögeln. Centralblatt für Bakteriologie und Parasitenkunde, 1891, 9, 403-409, 429-433, 461-467.

Gres V. \& LANDau I. Les Plasmodium de Lophura (Phasianidae): redescription de $P$. lophurae Coggeshall, 1938 et description de deux nouvelles espèces. Zoosystema, 1997 , 19, 545-555.

Grulet O., Landau I. \& Baccam D. Les Isospora du Moineau domestique; multiplicité des espèces. Annales de Parasitologie Humaine Comparée, 1982, 57 (3), 209-235.

Hawking F., Worms M.E. \& Gammage K. Duration of the mature gametocytes of $P$. gallinaceum. Transactions of the Royal Society of Tropical Medecine and Hygiene, 1969, 63, 421.

Huff C.G. Plasmodium elongatum n. sp., an avian malarial organism with an elongated gametocyte. American Journal of Hygiene, 1930, 11, 385-391.

Huff C.G. Plasmodium hexamerium, n. sp. from the BlueBird, inoculable to canaries. American Journal of Hygiene, 1935, 22, 274-277.

Kiкuth W. Immunobiologische und Chemotherapeutische Studien an Verschiedenen Stämmen von Vogelmalaria. Zentralblatt für Bakteriologie, Parasitenkunde, Infektionskrankbeiten und Hygiene I, Abt., Orig., 1931, 121, 401409.

Landau I., Chabaud A.G., Bertani S. \& Snounou G. Taxonomic status and re-description of Plasmodium relictum (Grassi et Feletti, 1891), Plasmodium maior, Raffaele, 1931 and description of P. bigueti n. sp. in sparrows. Parassitologia, 2003, 45, 119-123.

MANwELL R.D. How many species of avian malaria parasites are there? Journal of Parasitology, 1934, 20, 334.

MANWELl R.D. How many species of avian malaria parasites are there? American Journal of Tropical Medecine, 1935, $15,265-283$

Manwell R.D. Plasmodium octamerium n. sp., an avian malaria parasite from the Pintail Whydah Bird Vidua macroura. Journal of Protozoology, 1968, 15 (4), 680685.

Manwell R.D. \& Kuntz R.E. A new species of Plasmodium from the Formosan Shoveller Duck. Journal of Protozoology, 1965, 12 (1), 101-104.

Manwell R.D. \& Kuntz R.E. Plasmodium hegneri n. sp. from the European Teal Anas c. crecca in Taiwan. Journal of Protozoology, 1966, 13 (3), 437-440

PetTer A.J. Equilibre des espèces dans les populations de Nématodes parasites du colon des Tortues terrestres. Mémoires du Muséum National d'Histoire Naturelle., Nouvelle Série A, Zoologie, 1966, 39, 1-252.

RAfFaele G. Osservazioni sui plasmodidi degli uccelli. Rivista di Malariologia, 1930, 9 (3), 209-218 + 1 pl.

Rageau J., Mouchet J. \& AbBonnenc E. Répartition géographique des moustiques (Dipera : Culicida) en France. Cabiers ORSTOM série Entomologie Médicale et Parasitologie, 1970, 8 (3), 289-318. 
SCHAFFNER F. A revised checklist of French mosquitoes. European Mosquito Bulletin, 1998, 2, 1-9.

SCHwetz J. Sur un Plasmodium aviaire à formes de division allongées, Plasmodium fallax, n. sp. Archives de l'Institut Pasteur d'Algérie, 1930, 8, 289-296.

Sergent E., Sergent E. \& Catanei A. Sur un parasite nouveau du paludisme des oiseaux. Comptes Rendus Hebdomadaires des Séances de l'Académie des Sciences, Paris, 1928, 186, 809-811.

ValkiÛNas G. Avian Malaria Parasites and other Haemosporidia. CRC Press, Boca Raton, Florida, 2005, pp. 934.

Reçu le 19 septembre 2006 Accepté le 14 novembre 2006 